\title{
PION NUCLEON SCATTERING: SOME RESULTS FROM LATTICE QCD
}

\author{
C. B. LANG2 ${ }^{+}$and V. VERDUCI ${ }^{+, \#}$ \\ +Institut für Physik, Universität Graz, A-8010 Graz, Austria \\ \#School of Physics and Astronomy, University of Edinburgh, \\ Edinburgh EH9 3JZ, Scotland
}

\begin{abstract}
Including the meson-baryon (5 quark) intermediate states in a lattice simulation is challenging. However, it is important in order to obtain the correct energy eigenstates and to relate them to scattering phase shifts. Recent results for the negative parity nucleon channel and the problem of baryonic resonances in lattice calculations are discussed.
\end{abstract}

\section{Motivation}

The Euclidean space-time lattice regularisation is the only controllable nonperturbative regularisation of QCD. It defines the quantum field theory as a limit from a finite to an infinite number of points and from non-zero to vanishing lattice spacing. At any step in the limit one can in principle solve the functional integrals and determine values for $n$-point amplitudes by numeric means. The last decades have shown an enormous progress towards computing hadronic properties that way, mainly by Monte Carlo methods on very large computers.

Due to the finiteness of the spatial volume, correlation functions of the type $\langle\mathcal{O}(t) \mathcal{O}(0)\rangle$ have a discrete energy spectrum - as opposed to the continuous spectral function in the continuum. The ground states can be computed from the correlation function using hadronic operators in the corresponding quantum channel.

In Nature the hadrons are, however, stable (w.r.t. hadronic decays) only in a few cases (proton, pion, kaon,...). In the lattice world, where the quark mass may be set to unphysically large values and where the momenta are

\footnotetext{
${ }^{1}$ Contribution to the Nstar 2013 Workshop, Peniscola, Spain, May 27-30, 2013

${ }^{2}$ Speaker at the conference
} 
quantized due to the finite spatial volume, some decay thresholds lie higher and the respective lowest state appears to be (artificially) stable. On the other hand, two- (and more-) hadron intermediate states should have an impact on the energy spectrum. Most of those have not been observed up to now. In recent years there have been several studies determining baryonic excitations. [1, 2, 3, 4 $]^{3}$ One common feature was the absence of meson-baryon decay states (except for possibly the $s$-wave states, see below). This was especially blatant in the $\rho \rightarrow \rho$ studies, which on the lattice should exhibit $p$ wave $\pi \pi$ intermediate states. These should show up (but did not) as discrete energy levels of the spectral decomposition of the hadron correlation function. In the meson sector the cure was to include meson-meson interpolators in the set of operators of the correlation matrix. Once these were allowed for, the diagonalization of the cross-correlation matrix exhibited the levels that had been missing before. The earlier observed levels, that had been interpreted as resonance excitations appeared shifted, depending on resonance width and lattice volume.

In the mentioned baryon studies, the baryon interpolators were exclusively three-quark operators and the statements above applied. Possible exceptions may be $s$-wave states like the negative parity nucleon $N\left(\frac{1}{2}^{-}\right)$where recent results indicated a coupling the $N \pi$ even when using only three-quarks correlators. [2, 3, 4]

In order to clarify the situation we studied the negative parity nucleon channel including $s$-wave $N \pi(4+1$ quark) operators. [5] Indeed we find significant differences in the energy spectrum, as will be discussed below. Our study is for gauge configurations with two mass degenerate dynamical quarks, generously provided by the authors of Ref. [6]. The used parameters correspond to a lattice spacing of $0.1239 \mathrm{fm}$ on a $16^{3} \times 32$ lattice with a pion mass of $266 \mathrm{MeV}$. Details can be found in Ref. [5].

\section{Tools}

Operators: For determining the low lying energy levels we applied the so-called variational analysis. One computes the cross-correlation matrix between a sufficiently complete set of hadronic lattice operators with the

\footnotetext{
${ }^{3}$ For brevity we omit the references to the equally important detailed studies on meson excitations.
} 

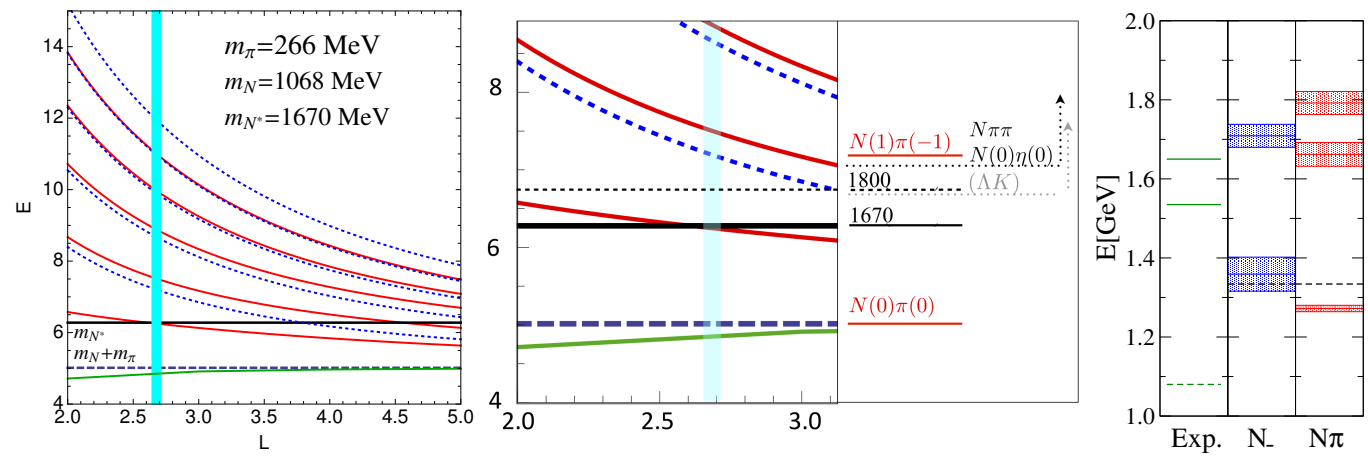

Figure 1: The $N \pi$ levels without (blue) and with (red and green) interaction assuming a Breit-Wigner shape phase shift for our situation. All energies as well as the spatial size $L$ use units of $m_{\pi}=266 \mathrm{MeV}$. The lowest $N \pi$ level approaches the threshold from below towards $L \rightarrow \infty$. Middle: Details, possible coupled channels thresholds are indicated; note that the numbers 1670 and 1800 correspond to the $N^{*}$ resonances in a world with $m_{\pi}=266 \mathrm{MeV}$, thus they come out higher than their physical values. R.h.s.: Comparison of the energy levels observed without (middle) and with (right) including $N \pi$ operators.

chosen quantum numbers $\left(J^{P}=\frac{1}{2}^{-}\right.$in our case $)$. For the nucleon interpolator we use the standard operator (on a given time slice)

$$
\left(N_{ \pm}^{(i)}\right)_{\mu}(\vec{p}=0)=\sum_{\vec{x}} \epsilon_{a b c}\left(P_{ \pm} \Gamma_{1}^{(i)} u_{a}(\vec{x})\right)_{\mu}\left(u_{b}^{T}(\vec{x}) \Gamma_{2}^{(i)} d_{c}(\vec{x})\right)
$$

$\left(\Gamma_{1}, \Gamma_{2}\right)$ can assume the three values $\left(\mathbb{1}, C \gamma_{5}\right),\left(\gamma_{5}, C\right)$ and (i1, $\left.C \gamma_{t} \gamma_{5}\right)$ for $i=1,2,3$. $C$ denotes the charge conjugation matrix, $\gamma_{t}$ the Dirac matrix in time direction, and $P_{ \pm}=\frac{1}{2}\left(1 \pm \gamma_{t}\right)$ the parity projection. We sum over all points of the time slice in order to project to zero momentum. The pion interpolator is, e.g., $\pi^{+}(\vec{p}=0)=\sum_{\vec{x}} \bar{d}_{a}(\vec{x}) \gamma_{5} u_{a}(\vec{x})$. Summation over the color index $a$ is implied.

For the $N \pi$ system in the rest frame the leading $s$-wave contribution comes from the interpolator with both particles at rest, $N \pi(\vec{p}=0)=$ $\gamma_{5} N_{+}(\vec{p}=0) \pi(\vec{p}=0)$, where $N_{+}$denotes the positive parity nucleon and the factor $\gamma_{5}$ ensures negative parity for the interpolator. We project to isospin $\frac{1}{2}$. In the distillation approach we choose $N_{v}=32$ and 64 for each of the 3 quark nucleon interpolators and $N_{v}=32$ for the $N \pi$ channel. We then select 
the most suitable combination and diagonalize a $7 \times 7$ correlation matrix to obtain the results shown in Fig. 1.

Variational analysis: We determine the energy levels of the coupled $N$ and $N \pi$ system with help of the variational method. [7] For a given quantum channel one measures the Euclidean cross-correlation matrix $C(t)$ between several interpolators living on the corresponding Euclidean time slices. The generalized eigenvalue problem disentangles the eigenstates $|n\rangle$ and from the exponential decay of the eigenvalues one determines the energy values of the eigenstates by exponential fits to the asymptotic behavior.

Wick contractions: In order to compute this correlation matrix we had to first compute the Wick decomposition of the correlators in terms of the quark propagators. For $N \rightarrow N$ these are of two types, while the complete $\left(N_{-}, N_{+} \pi\right) \leftrightarrow\left(N_{-}, N_{+} \pi\right)$ system requires the evaluation of 29 graphs. [5]

Distillation: Among all these contraction terms, there are some involving backtracking propagators where special tools are necessary to obtain statistically reliable signals. We used the so-called distillation method. [8] On a given time slice one introduces separable quark smearing sources derived from the eigenvectors of the spatial lattice Laplacian. This allows high flexibility due to the disentanglement of the computation of the quark propagators ("perambulators") and the hadron operators.

Phase shifts: Lüscher [9] derived a relation between the energy levels at finite volume and the phase shifts of the infinite volume, valid in the elastic region. For the pion mass used, the nucleon mass lies roughly $130 \mathrm{MeV}$ above the physical one. Similar mass shifts occur for other resonances. We argue (see Fig. 1), that the thresholds for $N \eta, N \pi \pi$ and $\Lambda K$ lie (closely) above the two lowest states $(N(1535)$ and $N(1650)$, shifted upwards as well), i.e., above $1800 \mathrm{MeV}$. Also the $N(1) \pi(-1)$ state (the value " 1 " refers to the smallest momentum unit $2 \pi / 16 a$ for our spatial lattice extent) lies higher. We did not include these meson-baryon operators in our set. Thus we apply Lüscher's relation up to the region of the second resonance. More detailed studies would have to include more operators and deal with the coupled channel problem. For a discussion of pole shifting due to changes in the mass see Ref. [10].

\section{Results}

Fig. 11 (r.h.s.) demonstrates that the energy spectrum changes when allowing for the $N \pi$ coupled channel. We find now a clear signal for the lowest 
$N(0) \pi(0)$ state, lying closely below threshold, as expected. Indeed also the quality of the energy levels improves. In a Breit-Wigner fit to the corresponding phase shift values the resonance masses lie approximately $150 \mathrm{MeV}$ above the physical values, similar to the nucleon mass, due to the unphysical pion mass of $266 \mathrm{MeV}$. Further details can be found in Refs. [5] and [11].

\section{Acknowledgements}

We thank Michael Döring, Georg Engel, Christof Gattringer, Leonid Glozman, Meinulf Göckeler, Daniel Mohler, Colin Morningstar, Sasa Prelovsek and Akaki Rusetsky for many discussions. Special thanks to Anna Hasenfratz for providing the dynamical configurations and to Daniel Mohler and Sasa Prelovsek for allowing us to use the perambulators derived in another project. V.V. has been supported by the Austrian Science Fund (FWF) under Grant No. DK W1203-N16.

\section{References}

[1] R. G. Edwards, J. J. Dudek, D. G. Richards, and S. J. Wallace, Phys. Rev. D 84, 074508 (2011).

[2] M. S. Mahbub, W. Kamleh, D. B. Leinweber, P. J. Moran, and A. G. Williams, Phys. Rev. D87, 011501 (2013).

[3] G. P. Engel, C. B. Lang, D. Mohler, and A. Schaefer, Phys. Rev. D87, 074504 (2013).

[4] C. Alexandrou, T. Korzec, G. Koutsou, and T. Leontiou, Nucleon Excited States in $N_{f}=2$ lattice QCD, (2013), arXiv:1302.4410.

[5] C. B. Lang and V. Verduci, Phys. Rev. D87, 054502 (2013).

[6] A. Hasenfratz, R. Hoffmann, and S. Schaefer, Phys. Rev. D 78, 054511 (2008): ibid. D 78, 014515 (2008).

[7] C. Michael, Nucl. Phys. B 259, 58 (1985). M. Lüscher and U. Wolff, Nucl. Phys. B 339, 222 (1990). B. Blossier, M. DellaMorte, G. von Hippel, T. Mendes, and R. Sommer, JHEP 0904, 094 (2009). 
[8] Hadron Spectrum Collaboration, M. Peardon et al., Phys. Rev. D 80, 054506 (2009).

[9] M. Lüscher, Commun. Math. Phys. 104, 177 (1986), ibid. 105, 153 (1986); Nucl. Phys. B 354, 531 (1991); Nucl. Phys. B 364, 237 (1991).

[10] M. Döring, M. Mai, and U.-G. Meißner, Phys. Lett. B 722, 185 (2013).

[11] C. B. Lang and V. Verduci, Nucleon excited states on the lattice, (2013), arXiv:1304.4114. 